\title{
The evolution of the cosmic microwave background temperature ${ }^{\star}$ Measurements of $T_{\mathrm{CMB}}$ at high redshift from carbon monoxide excitation
}

\author{
P. Noterdaeme ${ }^{1}$, P. Petitjean ${ }^{2}$, R. Srianand ${ }^{3}$, C. Ledoux ${ }^{4}$, and S. López ${ }^{1}$ \\ ${ }^{1}$ Departamento de Astronomía, Universidad de Chile, Casilla 36-D, Santiago, Chile \\ e-mail: [pasquier; slopez]@das.uchile.cl \\ 2 Université Paris 6, Institut d'Astrophysique de Paris, CNRS UMR 7095, 98bis bd Arago, 75014 Paris, France \\ e-mail: petitjean@iap.fr \\ 3 Inter-University Centre for Astronomy and Astrophysics, Post Bag 4, Ganeshkhind, 411007 Pune, India \\ e-mail: anand@iucaa.ernet.in \\ ${ }^{4}$ European Southern Observatory, Alonso de Córdova 3107, Vitacura, Casilla 19001, Santiago 19, Chile \\ e-mail: cledoux@eso.org
}

Received 15 November 2010 / Accepted 13 December 2010

ABSTRACT

\begin{abstract}
A milestone of modern cosmology was the prediction and serendipitous discovery of the cosmic microwave background (CMB), the radiation leftover after decoupling from matter in the early evolutionary stages of the Universe. A prediction of the standard hot Big-Bang model is the linear increase with redshift of the black-body temperature of the CMB $\left(T_{\mathrm{CMB}}\right)$. This radiation excites the rotational levels of some interstellar molecules, including carbon monoxide (CO), which can serve as cosmic thermometers. Using three new and two previously reported $\mathrm{CO}$ absorption-line systems detected in quasar spectra during a systematic survey carried out using VLT/UVES, we constrain the evolution of $T_{\mathrm{CMB}}$ to $z \sim 3$. Combining our precise measurements with previous constraints, we obtain $T_{\mathrm{CMB}}(z)=(2.725 \pm 0.002) \times(1+z)^{1-\beta} \mathrm{K}$ with $\beta=-0.007 \pm 0.027$, a more than two-fold improvement in precision. The measurements are consistent with the standard (i.e. adiabatic, $\beta=0$ ) Big-Bang model and provide a strong constraint on the effective equation of state of decaying dark energy (i.e. $w_{\text {eff }}=-0.996 \pm 0.025$ ).
\end{abstract}

Key words. cosmology: observations - cosmic background radiation - quasars: absorption lines

\section{Introduction}

The existence of the cosmic microwave background (CMB) radiation is a fundamental prediction of the hot Big-Bang theory. If gravitation is described by general relativity and electromagnetism by the Maxwell theory, then photons propagate along null geodesics and the CMB black-body temperature must follow the relation $T_{\mathrm{CMB}}(z)=T_{\mathrm{CMB}}^{0} \times(1+z)^{1-\beta}$, with $\beta=0$ and where $T_{\mathrm{CMB}}^{0}=2.725 \pm 0.002 \mathrm{~K}$ (Mather et al. 1999) is the temperature measured locally (at redshift $z=0$ ). This relation, which is a theoretical consequence of the adiabatic expansion of the Universe, needs to be verified by direct measurements. This has deeper theoretical implications as well (Uzan et al. 2004). A non-zero $\beta$ would indicate either a violation of the hypothesis of local position invariance (and thus of the equivalence principle) or that the number of photons is not conserved - with the constraint that the energy injection does not induce spectral distortion of the CMB. In the first case, this should be associated with a variation of the fundamental constants (see e.g. Murphy et al. 2003; Srianand et al. 2004).

There are currently two methods to measure $T_{\mathrm{CMB}}$ at redshifts $z>0$. The first one relies on the measurement of a small change in the spectral intensity of the CMB towards clusters

^ Based on observations carried out at the European Southern Observatory (ESO) using the Ultraviolet and Visual Echelle Spectrograph (UVES) at the Very Large Telescope (VLT, UT2-Kueyen) under Prgm. IDs 278.A-5062(A), 081.A-0334(B), 082.A-0544(A), and 083.A-0454(A). of galaxies owing to inverse Compton scattering of photons by the hot intra-cluster gas: the so-called Sunyaev-Zel'dovich (S-Z) effect (Fabbri et al. 1978; Rephaeli 1980). Although this technique permits precise measurements $(\Delta T \sim 0.3 \mathrm{~K}$; Battistelli et al. 2002; Luzzi et al. 2009), the method is essentially limited to $z<0.6$ because of the scarcity of known clusters at higher redshifts. The other technique uses the excitation of interstellar atomic or molecular species that have transition energies in the sub-millimetre range and can be excited by CMB photons. When the relative population of the different energy levels are in radiative equilibrium with the $\mathrm{CMB}$ radiation, the excitation temperature of the species equals that of the black-body radiation at that redshift. Therefore, the detection of these species in diffuse gas, where collisional excitation is negligible, provides one of the best thermometers for determining the black-body temperature of the CMB in the distant Universe (Bahcall \& Wolf 1968).

\section{Constraining $T_{\mathrm{CMB}}(z)$ using QSO absorption systems}

The observation of diffuse gas at high redshift is routinely achieved through the absorption lines that the interstellar medium of high- $z$ galaxies produces in the spectra of bright background sources such as quasars. However, detecting suitable species for measuring $T_{\mathrm{CMB}}$ turns out to be difficult.

\subsection{Atomic species}

Several attempts to measure $T_{\mathrm{CMB}}$ at high redshift have been made using the excitation of fine-structure levels of atomic 
species like carbon. These measurements generally led to upperlimits on $T_{\mathrm{CMB}}$ because collisional excitation dominates (e.g. Meyer et al. 1986; Lu et al. 1996; Roth \& Bauer 1999; Ge et al. 2001). Assuming the CMB is the only source of excitation, Songaila et al. (1994) measured $T_{\mathrm{CMB}}=7.4 \pm 0.8 \mathrm{~K}$ from $\mathrm{C}^{0}$ at $z=1.776$ towards the quasar Q $1331+170$. Cui et al. (2005) later derived the physical conditions in the same system through the observation of molecular hydrogen (see also Carswell et al. 2010). Applying the inferred physical conditions to the excitation of $\mathrm{C}^{0}$, they got $T_{\mathrm{CMB}}=7.2 \pm 0.8 \mathrm{~K}$. Similarly, Ge et al. (1997) estimated $T_{\mathrm{CMB}}=7.9 \pm 1.0 \mathrm{~K}$ at $z=1.9731$ from the excitation of $C^{0}$ towards Q 0013-004. Srianand et al. (2000) performed a detailed study of the physical conditions in the $\mathrm{H}_{2}$-bearing system towards PKS $1232+0815$, which allowed for both stringent lower $(>6 \mathrm{~K})$ and upper $(<14 \mathrm{~K})$ limits on $T_{\mathrm{CMB}}$ at $z=2.3377$. Finally, Molaro et al. (2002) used $\mathrm{C}^{+}$to constrain $T_{\mathrm{CMB}}=12.1_{-3.2}^{+1.7} \mathrm{~K}$ at $z=3.025$ towards Q 0347-3819. In all these cases, collisional excitation was not negligible and had to be corrected for, introducing uncertainties in the measurement. Taking a conservative approach, Srianand et al. (2008) assumed the CMB to be the only source of excitation to provide stringent upper-limits on $T_{\mathrm{CMB}}$ for a large sample of $\mathrm{C}$ I absorption lines detected in high signal-to-noise, high-resolution spectra.

Other systematic effects may also be hard to control. For example, the accuracy of the oscillator strengths of $\mathrm{C}^{0}$ finestructure levels (e.g. Jenkins \& Tripp 2001; Morton 2003) is subject to discussions. It is therefore important to find a direct and independent way of measuring $T_{\mathrm{CMB}}$ at similar redshifts.

\subsection{Molecular species}

The rotational excitation of molecules with permanent dipolar momentum can provide a more direct and precise measurement. For instance, the $\mathrm{CN}$ molecule has proved to be a remarkable thermometer of the CMB in the Galaxy and has been used for precise measurement of $T_{\mathrm{CMB}}$ in different directions (e.g. Meyer \& Jura 1985; Kaiser \& Wright 1990; Roth et al. 1993; Ritchey et al. 2010). However, up to now, $\mathrm{CN}$ has not been detected in diffuse gas at high redshift. On the other hand, an exciting possibility is offered by carbon monoxide (CO), whose energy differences between rotational levels are close to $k T_{\mathrm{CMB}}$ at high redshift. The use of $\mathrm{CO}$ in this kind of experiments was prevented because $\mathrm{CO}$ has been detected in absorption only very recently at high- $z$ (Srianand et al. 2008; Noterdaeme et al. 2009, 2010). Indeed, the detection of $\mathrm{CO}$ in diffuse gas at high redshift is challenging because of the low dust opacity required to observe the background source. Because shielding from the surrounding UV radiation field becomes more efficient deeper inside an interstellar cloud, carbon is found predominantly in its ionised $\left(\mathrm{C}^{+}\right)$, atomic $\left(\mathrm{C}^{0}\right)$, and molecular forms $(\mathrm{CO})$ in the external, intermediate, and deepest layers of the cloud, respectively. Since the lines of sight passing through the central and fully molecular part of the cloud are affected by strong extinction, we targeted the $\mathrm{C}^{0}$ phase so that the light from the corresponding background quasar is only moderately obscured by the diffuse molecular gas, while some $\mathrm{CO}$ is present in the gas.

\section{Candidate search, follow-up, and analysis}

We have searched over 40000 quasar spectra from the Sloan Digital Sky Survey data base (SDSS-DR7, Abazajian et al. 2009) for strong intervening $\mathrm{C}$ I absorption lines that can be detected even in low-resolution spectra. This led to the detection of 67 $\mathrm{C}^{0}$-bearing absorption systems at redshifts between 1.5 and 3 . We note that no pre-selection was made upon the presence of neutral atomic hydrogen, i.e., the systems did not need to be damped Lyman- $\alpha$ systems ${ }^{1}$ (DLAs, see Wolfe et al. 2005). Indeed, the Lyman- $\alpha$ line is covered by the SDSS spectra only for $z_{\mathrm{abs}} \geq 2.2$, while $\mathrm{C}$ I can be detected down to $z_{\mathrm{abs}} \sim 1.5$.

Because $\mathrm{CO}$ absorption lines are expected to be weak and not detectable at the SDSS spectral resolution $(\lambda / \Delta \lambda \simeq 2000)$, the most promising candidates were subsequently re-observed at a resolving power of $\lambda / \Delta \lambda \simeq 50000$ with the Ultraviolet and Visual Echelle Spectrograph (UVES), mounted on the ESO Very Large Telescope. We present here three new detections of $\mathrm{CO}$ for which we derive the rotational excitation temperatures. Adding two previous measurements using $\mathrm{CO}$ by our group (Srianand et al. 2008; Noterdaeme et al. 2010) ${ }^{2}$, we constrain the CMB black-body temperature at redshifts $1.7<z<2.7$. Each quasar line-of-sight was observed for a total of three to nine hours with UVES. The data were reduced using the UVES pipeline, and individual spectra were shifted to the heliocentric-vacuum frame and combined by weighting each pixel by the inverse of the associated variance.

The CO molecule possesses rotational, vibrational, and electronic transitions that correspond to photons with radio, infrared, and far-ultraviolet wavelengths, respectively. We detect several absorption bands from the electronic transitions of $\mathrm{CO}$ in the vibrational ground-state, redshifted into the optical wavelength range. Each electronic band is split into three branches composed of several absorption lines arising from different rotational levels. These absorption lines are resolved in the UVES data, and the excitation temperatures of $\mathrm{CO}$ can be precisely measured thanks to the large number of lines (for each rotational level and spanning a range of oscillator strengths) that are fitted simultaneously.

The CO profiles were modelled with multiple Voigt profiles using a $\chi^{2}$-minimisation technique. The wavelengths and oscillator strengths were taken from Morton \& Noreau (1994). For three of the five CO-bearing systems presented here, the profiles are well resolved and the signal-to-noise ratio obtained is sufficiently high to measure the column densities in rotational levels up to $J=3$ independently. The excitation temperature is then measured by modelling the rotational level populations with a single Boltzmann distribution as shown in the right-hand side panel of Fig. 1. The detailed analysis of the systems towards SDSS J143912+111740 and SDSS J123714+064759 were presented in Srianand et al. (2008) and Noterdaeme et al. (2010), respectively. We used the same Voigt-profile fitting techniques to analyse the system towards SDSS J104705+205734 (see Fig. 1). A single velocity component was used to model the $\mathrm{CO}$ profile, leaving the column density in each rotational level, the Doppler parameter $b$, and the redshift of the CO-bearing cloud free. All absorption lines from the AX (0-0) to the AX (4-0) band were fitted simultaneously, while the AX (5-0) band was ignored during the fitting process because of a clear blending. We obtained $\log N(\mathrm{CO})=14.74 \pm 0.07, b=1.1 \pm 0.1 \mathrm{~km} \mathrm{~s}^{-1}$ and $T_{\text {ex }}(\mathrm{CO})=7.8_{-0.6}^{+0.7} \mathrm{~K}$. For the remaining two systems (towards SDSS J085726+185524 and SDSS J170542+354340), the rotational levels lines are hardly resolved ${ }^{3}$ and the signal-to-noise

\footnotetext{
1 Indeed, several systems turn out to be sub-DLAs (i.e. with $N\left(\mathrm{H}^{0}\right)<$ $2 \times 10^{20} \mathrm{~cm}^{-2}$ ).

2 Note that we detected a sixth CO-bearing system (at $z=1.64$ towards SDSS J160457.50+220300.5, Noterdaeme et al. 2009). However, this system is not suitable for constraining $T_{\mathrm{CMB}}$ because of the low $\mathrm{S} / \mathrm{N}$ ratio achieved and the complexity of the absorption profile.

3 This is mainly because of larger Doppler parameters in these systems ( $b \sim 5 \mathrm{~km} \mathrm{~s}^{-1}$ ) similar to those of the S I lines associated to the $\mathrm{CO}$ components.
} 
P. Noterdaeme et al.: The evolution of the cosmic microwave background temperature
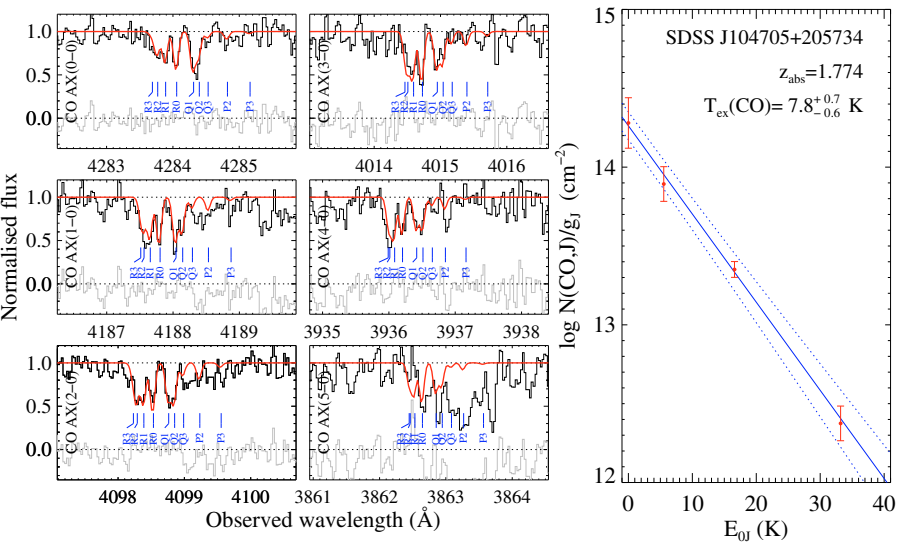

Fig. 1. Left: $A^{1} \Pi\left(v^{\prime}\right) \leftarrow X^{1} \Sigma^{+}(v=0)$ electronic bands of $\mathrm{CO}$ detected at $z_{\mathrm{abs}}=1.774$ towards the quasar SDSS J104705+205734. The modelled profile is over-plotted in red with the fitting residuals shown in grey. Short vertical lines indicate the branch ("R", "P" or "Q") and the rotational level of the transition $(J=0-3)$. Right: the corresponding excitation diagram of $\mathrm{CO}$ gives the column density of the rotational levels $J$ weighted by their quantum degeneracy $\left(g_{J}\right)$ versus the energy of these levels relative to the ground state. The excitation temperature is directly given by $-1 /(a \ln 10)$ where $a$ is the slope of the linear fit (solid blue line, with $1 \sigma$ errors represented by the dotted curves).
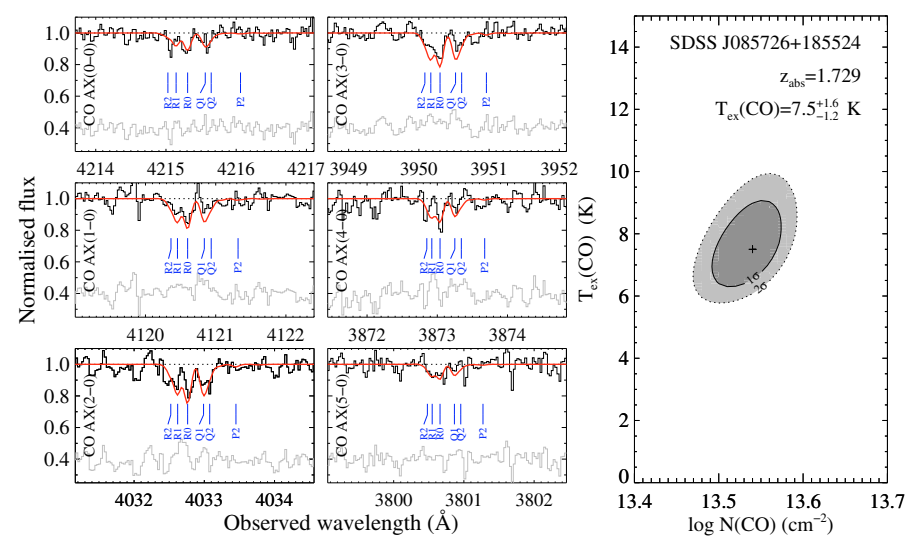

Fig. 2. Left: the CO electronic absorptions from the system at $z_{\text {abs }}=$ 1.729 towards SDSS J085726+185524 are plotted together with the best-fit model $\left(\log N=13.54, T_{\mathrm{ex}}=7.5 \mathrm{~K}\right.$, red profile). Right: confidence contours for the excitation temperature vs the total CO column density.

ratio is not high enough to measure the column densities in each rotational level independently. Instead, we used a technique similar to that presented in Burgh et al. (2007) and Prochaska et al. (2009): a single excitation temperature was used as an external parameter to model the $\mathrm{CO}$ profile directly, again, using a Boltzmann distribution of the rotational level populations. The best fit-model was chosen from the minimum $\chi^{2}$ obtained for a grid of total column densities and excitation temperatures (see right-hand side panels of Figs. 2 and 3).

The excitation temperatures for the five CO-bearing systems are given in Table 1. At high redshift, the excitation of $\mathrm{CO}$ is dominated by radiative excitation as predicted in diffuse interstellar clouds (Warin et al. 1996; Burgh et al. 2007). Indeed, the excitation temperatures we measured are well above the mean temperature measured in the Galaxy for similar CO column densities $\left(\left\langle T_{\text {ex }}\right\rangle=3.6 \mathrm{~K}\right.$, see Burgh et al. 2007). This is further supported by the low volume density of the gas derived from the analysis of $\mathrm{H}_{2}$ and $\mathrm{C}^{0}$ lines in two of these high- $z$ absorption systems (Srianand et al. 2008; Noterdaeme et al. 2010).

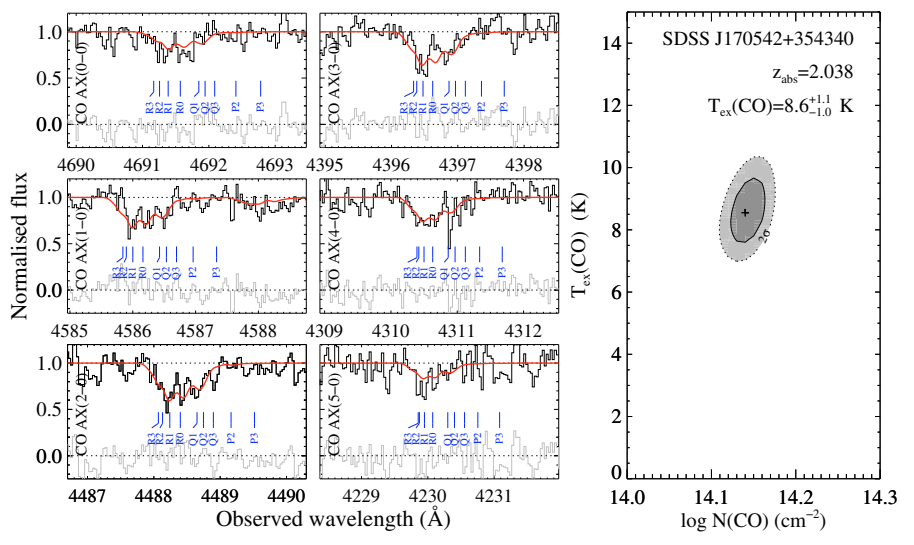

Fig. 3. Same as Fig. 2 for the system at $z_{\text {abs }}=2.038$ towards SDSS J170542+354340 (best-fit model: $\log N=14.14, T_{\text {ex }}=8.6 \mathrm{~K}$ ).

Table 1. CO excitation temperatures.

\begin{tabular}{cccc}
\hline \hline Quasar & $z_{\text {abs }}$ & $T_{\text {ex }}(\mathrm{CO})(\mathrm{K})$ & Ref. \\
\hline SDSS J085726+185524 & 1.7293 & $7.5_{-1.2}^{+1.6}$ & 1 \\
SDSS J104705+205734 & 1.7738 & $7.8_{-0.6}^{+0.7}$ & 1 \\
SDSS J123714+064759 & 2.6896 & $10.5_{-0.6}^{+0.8}$ & 2 \\
SDSS J143912+111740 & 2.4184 & $9.15_{-0.7}^{+0.7}$ & 3 \\
SDSS J170542+354340 & 2.0377 & $8.6_{-1.0}^{+1.1}$ & 1 \\
\hline
\end{tabular}

References. (1) this work; (2) Noterdaeme et al. (2010); (3) Srianand et al. (2008).

Therefore, $T_{\mathrm{ex}}(\mathrm{CO})$ must be a good proxy for $T_{\mathrm{CMB}}$ at high redshift.

\section{Conclusion}

The CMB temperature derived from the rotational excitation of $\mathrm{CO}$ in five absorption systems (three studied here plus two previously published, see Table 1) are presented in Fig. 4 together with measurements and upper-limits obtained from the analysis of the populations of the fine-structure energy levels of atomic carbon or from the S-Z effect in galaxy clusters. An upper-limit has also been obtained from the analysis of millimetre absorption lines from different molecules in the gravitational lens of PKS 1830-211 (Wiklind \& Combes 1996).

The technique presented here allows us to probe the temperature of the $\mathrm{CMB}$ at high redshift, providing constraints that are independent from and stronger than those arising from the analysis of $\mathrm{C}^{0}$ and $\mathrm{C}^{+}$. This demonstrates that the rotational excitation of interstellar $\mathrm{CO}$ molecules can provide a direct and precise measurement of $T_{\mathrm{CMB}}$ in the early Universe.

Fitting the measurements from different techniques with the expression $T_{\mathrm{CMB}}(z)=T_{\mathrm{CMB}}^{0} \times(1+z)^{1-\beta}($ Lima et al. 2000$)$, we get the constraints on $\beta$ summarised in Table 2. We note that combining $\mathrm{CO}$ measurements of $T_{\mathrm{CMB}}(z)$ with those obtained from other techniques improves the precision of the $\beta$-measurement by more than a factor of two. The measurement presented here, $\beta=-0.007 \pm 0.027$, directly supports the adiabatic evolution of the $\mathrm{CMB}$ radiation temperature $(\beta=0)$, expected from the standard hot Big-Bang model. Considering alternative $\Lambda$ cosmological models, Jetzer et al. (2010) demonstrated that measuring $T_{\mathrm{CMB}}$ at different redshifts allows one to constrain the effective equation of state of decaying dark energy $\left(p=w_{\text {eff }} \rho\right)$. Fitting the measurements of $T_{\mathrm{CMB}}$ with their temperature-redshift relation (Eq. (22) in Jetzer et al. 2010), taking $\Omega_{\mathrm{m}}=0.275 \pm 0.015$ 


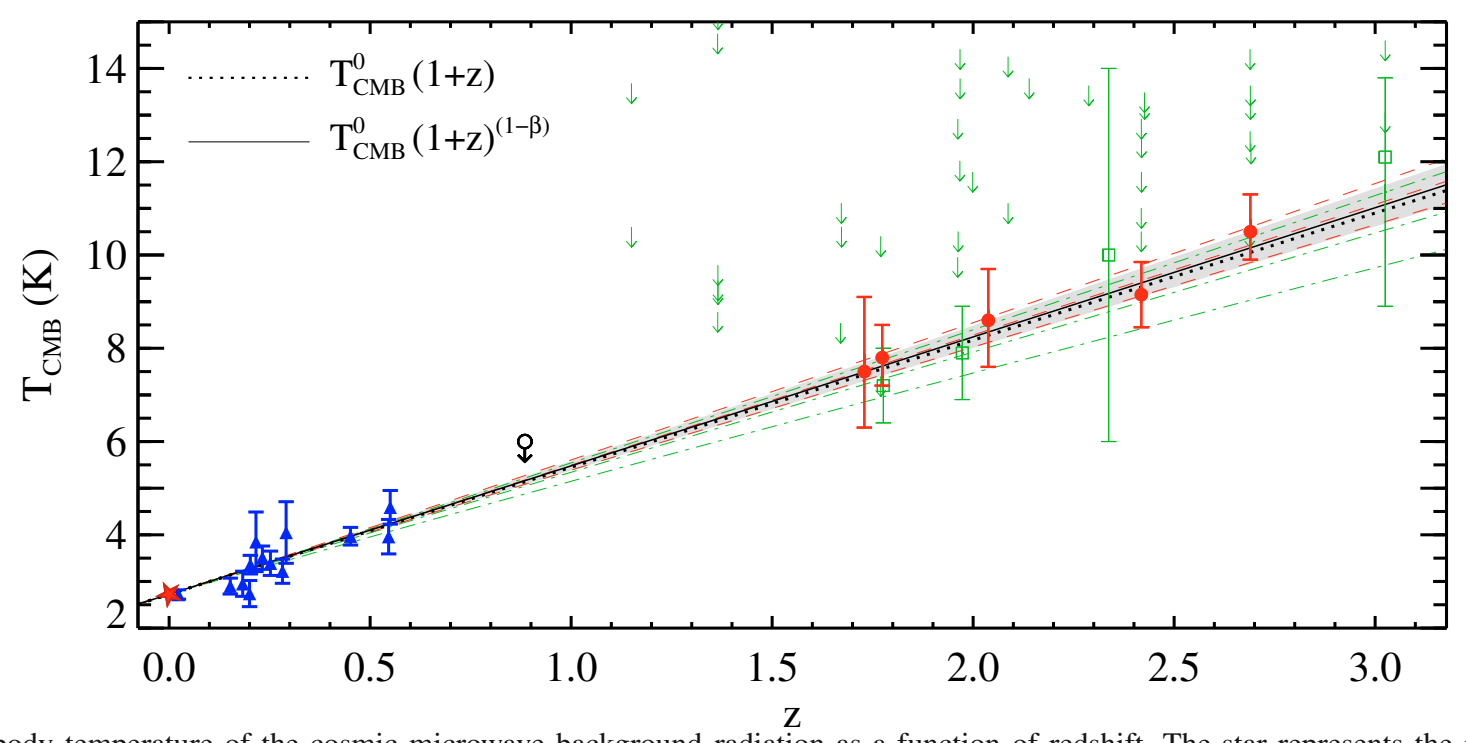

Fig. 4. Black-body temperature of the cosmic microwave background radiation as a function of redshift. The star represents the measurement at $z=0$ (Mather et al. 1999). Our measurements based on the rotational excitation of CO molecules are represented by red filled circles at $1.7<z<2.7$. Other measurements at $z>0$ are based (i) on the S-Z effect (blue triangles at $z<0.6$, Luzzi et al. 2009) and (ii) on the analysis of the fine structure of atomic carbon (green open squares: $z=1.8$, Cui et al. 2005; $z=2.0$, Ge et al. 1997; $z=2.3$, Srianand et al. 2000; $z=3.0$, Molaro et al. 2002). Upper limits come from the analysis of atomic carbon (from the literature and our UVES sample, see Srianand et al. 2008) and from the analysis of molecular absorption lines in the lensing galaxy of PKS 1830-211 (open circle at $z=0.9$, Wiklind \& Combes 1996). The dotted line represents the adiabatic evolution of $T_{\mathrm{CMB}}$ as expected in standard hot Big-Bang models. The solid line with shadowed errors is the fit using all the data and the alternative scaling of $T_{\mathrm{CMB}}(z)$ (Lima et al. 2000) yielding $\beta=-0.007 \pm 0.027$. The red dashed curve (resp. green dashed-dotted) represents the fit and errors using S-Z + CO measurements (resp. S-Z + atomic carbon).

Table 2. Constraints on the evolution of $T_{\mathrm{CMB}}$ with redshift.

\begin{tabular}{cc}
\hline \hline Data set & $\beta$ \\
\hline S-Z & $+0.040 \pm 0.079$ \\
S-Z + atom. carbon & $+0.029 \pm 0.053$ \\
S-Z + CO & $-0.012 \pm 0.029$ \\
S-Z + atom. carbon + CO & $-0.007 \pm 0.027$ \\
\hline
\end{tabular}

(Komatsu et al. 2010) and fixing $\gamma$ to the canonical value (4/3), we get $w_{\text {eff }}=-0.996 \pm 0.025$, which is a tighter constraint compared with those previously derived from other methods (e.g. Kowalski et al. 2008; Riess et al. 2009; Kessler et al. 2009; Jullo et al. 2010).

Finally, large and deep QSO surveys such as SDSS III should provide more lines of sight along which $\mathrm{CO}$ can be detected, while high-resolution spectrographs on future extremely large telescopes will allow for full de-blending of the CO lines in different rotational levels, which will in turn yield more accurate measurements.

Acknowledgements. We thank the referee, Paolo Molaro, for insightful comments that improved the paper. We are grateful to Jean-Philippe Uzan and Andrew Fox for their helpful comments on early versions of this paper. P.N. is a CONICYT/CNRS fellow and acknowledges the ESO Office for Science for hospitality and support. P.P.J. and R.S. acknowledge support from the Indo-French Centre for the Promotion of Advanced Research under programme N.4304-2.

\section{References}

Abazajian, K. N., Adelman-McCarthy, J. K., Agüeros, M. A., et al. 2009, ApJS, 182,543

Bahcall, J. N., \& Wolf, R. A. 1968, ApJ, 152, 701

Battistelli, E. S., De Petris, M., Lamagna, L., et al. 2002, ApJ, 580, L101

Burgh, E. B., France, K., \& McCandliss, S. R. 2007, ApJ, 658, 446

Carswell, R., Jorgenson, R., Wolfe, A., \& Murphy, M. 2011, MNRAS, accepted [arXiv: 1010.2821]

Cui, J., Bechtold, J., Ge, J., \& Meyer, D. M. 2005, ApJ, 633, 649

Fabbri, R., Melchiorri, F., \& Natale, V. 1978, Ap\&SS, 59, 223

Ge, J., Bechtold, J., \& Black, J. H. 1997, ApJ, 474, 67
Ge, J., Bechtold, J., \& Kulkarni, V. P. 2001, ApJ, 547, L1

Jenkins, E. B., \& Tripp, T. M. 2001, ApJS, 137, 297

Jetzer, P., Puy, D., Signore, M., \& Tortora, C. 2010, General Relativity and Gravitation, 191

Jullo, E., Natarajan, P., Kneib, J.-P., et al. 2010, Science, 329, 924

Kaiser, M. E., \& Wright, E. L. 1990, ApJ, 356, L1

Kessler, R., Becker, A. C., Cinabro, D., et al. 2009, ApJS, 185, 32

Komatsu, E., Smith, K. M., Dunkley, J., et al. 2011, ApJS, accepted [arXiv: 1001.4538$]$

Kowalski, M., Rubin, D., Aldering, G., et al. 2008, ApJ, 686, 749

Lima, J. A. S., Silva, A. I., \& Viegas, S. M. 2000, MNRAS, 312, 747

Lu, L., Sargent, W. L. W., Barlow, T. A., Churchill, C. W., \& Vogt, S. S. 1996, ApJS, 107, 475

Luzzi, G., Shimon, M., Lamagna, L., et al. 2009, ApJ, 705, 1122

Mather, J. C., Fixsen, D. J., Shafer, R. A., Mosier, C., \& Wilkinson, D. T. 1999, ApJ, 512, 511

Meyer, D. M., \& Jura, M. 1985, ApJ, 297, 119

Meyer, D. M., York, D. G., Black, J. H., Chaffee, Jr., F. H., \& Foltz, C. B. 1986, ApJ, 308, L37

Molaro, P., Levshakov, S. A., Dessauges-Zavadsky, M., \& D’Odorico, S. 2002, A\&A, 381, L64

Morton, D. C. 2003, ApJS, 149, 205

Morton, D. C., \& Noreau, L. 1994, ApJS, 95, 301

Murphy, M. T., Webb, J. K., \& Flambaum, V. V. 2003, MNRAS, 345, 609

Noterdaeme, P., Ledoux, C., Srianand, R., Petitjean, P., \& Lopez, S. 2009, A\&A, 503,765

Noterdaeme, P., Petitjean, P., Ledoux, C., et al. 2010, A\&A, 523, A80

Prochaska, J. X., Sheffer, Y., Perley, D. A., et al. 2009, ApJ, 691, L27

Rephaeli, Y. 1980, ApJ, 241, 858

Riess, A. G., Macri, L., Casertano, S., et al. 2009, ApJ, 699, 539

Ritchey, A. M., Federman, S. R., \& Lambert, D. L. 2011, ApJ, accepted [arXiv: 1012.1296]

Roth, K. C., \& Bauer, J. M. 1999, ApJ, 515, L57

Roth, K. C., Meyer, D. M., \& Hawkins, I. 1993, ApJ, 413, L67

Songaila, A., Cowie, L. L., Hogan, C. J., \& Rugers, M. 1994, Nature, 371, 43

Srianand, R., Chand, H., Petitjean, P., \& Aracil, B. 2004, Phys. Rev. Lett., 92, 121302

Srianand, R., Noterdaeme, P., Ledoux, C., \& Petitjean, P. 2008, A\&A, 482, L39

Srianand, R., Petitjean, P., \& Ledoux, C. 2000, Nature, 408, 931

Uzan, J., Aghanim, N., \& Mellier, Y. 2004, Phys. Rev. D, 70, 083533

Warin, S., Benayoun, J. J., \& Viala, Y. P. 1996, A\&A, 308, 535

Wiklind, T., \& Combes, F. 1996, Nature, 379, 139

Wolfe, A. M., Gawiser, E., \& Prochaska, J. X. 2005, ARA\&A, 43, 861 